\section{Clinical significance of RAS pathway alter- ations in pediatric acute myeloid leukemia}

\author{
Taeko Kaburagi, ${ }^{1,2}$ Genki Yamato, ${ }^{1,2}$ Norio Shiba, ${ }^{3}$ Kenichi Yoshida, ${ }^{4}$ Yusuke \\ Hara, ${ }^{2}$ Ken Tabuchi, ${ }^{5}$ Yuichi Shiraishi, ${ }^{6}$ Kentaro Ohki, ${ }^{7}$ Manabu Sotomatsu, ${ }^{1}$ \\ Hirokazu Arakawa, ${ }^{2}$ Hidemasa Matsuo, ${ }^{8}$ Akira Shimada, ${ }^{9}$ Tomohiko Taki, ${ }^{10}$ \\ Nobutaka Kiyokawa, ${ }^{7}$ Daisuke Tomizawa, ${ }^{11}$ Keizo Horibe,${ }^{12}$ Satoru Miyano, ${ }^{13}$ \\ Takashi Taga, ${ }^{14}$ Souichi Adachi, ${ }^{8}$ Seishi Ogawa ${ }^{4}$ and Yasuhide Hayashi ${ }^{1,15}$
}

${ }^{1}$ Department of Hematology/Oncology, Gunma Children's Medical Center, Gunma; ${ }^{2}$ Department of Pediatrics, Gunma University Graduate School of Medicine, Gunma; ${ }^{3}$ Department of Pediatrics, Yokohama City University Hospital, Kanagawa; ${ }^{4}$ Department of Pathology and Tumor Biology, Graduate School of Medicine, Kyoto University, Kyoto; ${ }^{5}$ Department of Pediatrics, Tokyo Metropolitan Cancer and Infectious Diseases Center Komagome Hospital, Tokyo; ${ }^{6}$ Division of Cellular Signaling, National Cancer Center Research Institute, Tokyo; 'Department of Pediatric Hematology and Oncology Research, National Research institute for Child Health and Development, Tokyo; ${ }^{8}$ Human Human Health Sciences, Graduate School of Medicine, Kyoto University, Kyoto; ${ }^{9}$ Department of Pediatrics, Okayama University, Okayama; ${ }^{10}$ Department or Medical Technology, Kyorin University Faculty of Health Sciences, Tokyo; ${ }^{11}$ Division of Leukemia and Lymphoma, Children's Cancer Center, National Center for Child Health and Development, Tokyo; ${ }^{12} \mathrm{Clinical}$ Research Center, National Hospital Organization Nagoya Medical Center, Aichi; ${ }^{13}$ Laboratory of Sequence Analysis, Human Genome Center, Institute of Medical Science, Tokyo University, Tokyo; ${ }^{14}$ Department of Pediatrics, Shiga University of Medical Science, Shiga and ${ }^{15}$ Institute of Physiology and Medicine, Jobu University, Gunma, Japan.

\section{ABSTRACT}

$\mathrm{R}$ AS pathway alterations have been implicated in the pathogenesis of various hematological malignancies. However, their clinical relevance in pediatric acute myeloid leukemia (AML) is not well characterized. We analyzed the frequency, clinical significance, and prognostic relevance of RAS pathway alterations in 328 pediatric patients with de novo AML. RAS pathway alterations were detected in $80(24.4 \%)$ of 328 patients: NF1 ( $\mathrm{n}=7,2.1 \%)$, PTPN11 ( $\mathrm{n}=15,4.6 \%), C B L(\mathrm{n}=6,1.8 \%)$, NRAS $(\mathrm{n}=44,13.4 \%), \operatorname{KRAS}(\mathrm{n}=12,3.7 \%)$. Most of these alterations in the RAS pathway were mutually exclusive also together with other aberrations of signal transduction pathways such as FLT3-ITD $(P=0.001)$ and KIT mutation $(P=0.004)$. NF1 alterations were frequently detected in patients with complex karyotype $(P=0.031)$ and were found to be independent predictors of poor overall survival $(O S)$ in multivariate analysis $(P=0.007)$. At least four of seven patients with NF1 alterations had biallelic inactivation. NRAS mutations were frequently observed in patients with $C B F B-M Y H 11$ and were independent predictors of favorable outcomes in multivariate analysis $(O S, P=0.023$; event-free survival [EFS], $P=0.037)$. Patients with PTPN11 mutations more frequently received stem cell transplantation $(P=0.035)$ and showed poor EFS than patients without PTPN11 mutations $(P=0.013)$. Detailed analysis of RAS pathway alterations may enable a more accurate prognostic stratification of pediatric AML and may provide novel therapeutic molecular targets related to this signal transduction pathway.

\section{Introduction}

Acute myeloid leukemia (AML) is characterized by considerable genetic heterogeneity. Several chromosomal aberrations and gene alterations have been identified in these patients; some of these have been found useful for risk stratification. ${ }^{1}$ Aberrations of signal transduction pathways (such as RAS family members, KIT, and FLT3) are considered as one of the most important pathogenetic factors in AML. ${ }^{2}$
Ferrata Storti Foundation

Haematologica 2022

Volume 107(3):583-592

\section{Correspondence:}

YASUHIDE HAYASHI

hayashiy@jobu.ac.jp

hayashiy-tky@umin.ac.jp

Received: August 28, 2020.

Accepted: March 12, 2021.

Pre-published: March 18, 2021.

https://doi.org/10.3324/haematol.2020.269431

(C)2022 Ferrata Storti Foundation

Material published in Haematologica is covered by copyright. All rights are reserved to the Ferrata Storti Foundation. Use of published material is allowed under the following terms and conditions:

https://creativecommons.org/licenses/by-nc/4.0/legalcode. Copies of published material are allowed for personal or internal use. Sharing published material for non-commercial purposes is subject to the following conditions:

https://creativecommons.org/licenses/by-nc/4.0/legalcode, sect. 3. Reproducing and sharing published material for commercial purposes is not allowed without permission in writing from the publisher. 
Recently, aberrations of NF1 and PTPN11 were reported to be associated with a poor prognosis in adult patients with AML. ${ }^{3,4}$ NF1 and PTPN11 are the family of RAS pathway genes and constitute the granulocyte-macrophage colony stimulating factor signaling pathway. Among the broad family of RAS pathway genes, mutations of $C B L$, NRAS and KRAS were also commonly detected in AML. ${ }^{2}$ These RAS pathway alterations have also been implicated in the causation of juvenile myelomonocytic leukemia (JMML). ${ }^{5}$

Mutations of PTPN11, NRAS, and KRAS have been reported in $3-4 \%, 6,7-13 \%, 6-11 \%$ of pediatric patients with AML, respectively. However, there is no clear consensus on the clinical significance of RAS pathway gene mutations especially NF1 and CBL mutations. ${ }^{10,11}$ The reported frequency of detection of CBL mutations and NF1 mutations or deletions in adult patients with AML is $0.6-0.7 \%$ and $3.5-10.5 \%,{ }^{14-16}$ respectively. However, the prognostic relevance of these mutations is not well characterized, particularly in pediatric AML patients.

In this study, we analyzed NF1, PTPN11, CBL, NRAS, and KRAS alterations in 328 pediatric patients with AML to determine the clinical significance of these alterations. We also examined the correlation of RAS pathway alterations with other genetic aberrations, cytogenetic alterations, and clinical characteristics.

\section{Methods}

\section{Patients}

Between November 2006 and December 2010, 443 pediatric patients with de novo AML (age $<18$ years) participated in the Japanese AML-05 trial conducted by the Japanese Pediatric Leukemia/Lymphoma Study Group (JPLSG). Treatment, data collection, and other details of the AML-05 study are presented in the Online Supplementary Appendix and the Online Supplementary Figure S1. This study was conducted in accordance with the Declaration of Helsinki and approved by the Institutional Review Board of the Gunma Children's Medical Center and the Ethical Review Board of the JPLSG.

\section{Mutation analysis of RAS pathway alterations}

We analyzed PTPN11 (exons 2-4, and 13), CBL (exons 8-9), $N R A S$ (exons 1-2), and KRAS (exons 1-2) mutations using Sanger sequencing as previously described. ${ }^{9,17,18}$ All coding exons of the NF1 were captured using the SureSelect custom kit (Agilent Technologies, Santa Clara, CA, USA), and sequenced using Hiseq 2500. Somatic mutations in NF1 were identified as described elsewhere. ${ }^{19}$

\section{Molecular characterization}

We analyzed KIT (exons 8, 10, and 17), ${ }^{20}$ NPM1 (exon 12), ${ }^{21}$ CEBPA (exons 1-4), ${ }^{22}$ CSF3R (exons 14 and 17), ${ }^{23}$ WT1 (exons 710), ${ }^{24}$ ASXL1 (exon 12), ASXL2 (exons 11 and 12), ${ }^{25}$ all exons of BCOR, BCORL1 ${ }^{26}$, RAD21, SMC3, STAG2, ${ }^{27}$ RUNX1, ${ }^{28}$ FLT3-ITD, ${ }^{29}$ and gene rearrangement of NUP98-NSD $1^{30}$ and FUS-ERG $G^{31}$ using Sanger sequencing. KMT2A-partial tandem duplication (PTD) was analyzed using the multiplex ligation-dependent probe amplification (MLPA) method. ${ }^{32}$ Quantitative real-time polymerase chain reaction (qRT-PCR) analysis of the PRDM16 and MECOM genes was performed using the 7900HT Fast Real Time PCR System, TaqMan Gene Expression Master Mix, and TaqMan Gene Expression Assay (Applied Biosystems, Foster City, CA, USA), as described elsewhere. ${ }^{33}$

\section{Copy number analysis}

Copy number $(\mathrm{CN})$ analysis was performed as previously reported $^{34}$ using an in-house pipeline CNACS (https://github.com/papaemmelab/toil_cnacs); the total number of reads covering each bait region and the allele frequency of heterozygous single-nucleotide polymorphisms (SNP) $(n=1,216)$ detected by targeted sequencing were used as input data. Based on the previous reports, ${ }^{15}$ we set the total $\mathrm{CN}<1.5$ as the definition of NF1 deletion.

\section{Statistical methods}

All statistical analyses were performed using the EZR software (version 1.35; Saitama Medical Center, Jichi Medical University, Saitama, Japan). ${ }^{35}$ Between-group differences with respect to clinical characteristics were assessed using the Fisher's exact and Mann-Whitney $U$ tests. Survival rates were estimated using the Kaplan-Meier method and compared using the log-rank test. Overall survival (OS) was defined as the time from diagnosis to death or last follow-up. Event-free survival (EFS) was defined as the time from diagnosis to the date of failure (induction failure, relapse, second malignancy, or death) for patients who experienced treatment failure or to the date of last contact for all other patients. Cox proportional hazards model was used to estimate hazard ratios and $95 \%$ Confidence Intervals (CI). For all analyses, two tailed $P$-values $<0.05$ were considered indicative of statistical significance.

\section{Results}

Frequencies of RAS pathway alterations in 328 pediatric acute myeloid leukemia patients

Out of the 443 patients, 115 patients were excluded from this study because of unavailability of genomic DNA samples. Therefore, 328 samples were analyzed in this study. We did not analyze germline alterations because of the lack of non-hematological or remission samples. The clinical characteristics of patients with available samples $(n=328)$ and those with no available samples $(n=115)$ are summarized in the Online Supplementary Table S1. White blood cell (WBC) count at diagnosis was significantly higher in the "sample available group" than in the "sample unavailable group" $(P<0.001)$. There were more patients who were at a low risk and there were less patients who were at an intermediate risk in the "sample available group" as compared with the "sample unavailable group" (low risk, $P=0.046$; intermediate risk, $P=0.003$ ). Cytogenetic features and prognosis were not significantly different between the available and unavailable samples (Online Supplementary Table S1).

RAS pathway alterations were detected in 80 (24.4\%) of the 328 patients; most of these alterations were mutually exclusive (Figure 1). The mutation sites and clinical characteristics of patients with RAS pathway alterations are summarized in Figure 2, Tables 1 and 2 and the Online Supplementary Tables S2 and S3, respectively.

We detected six NF1 mutations in four patients; all of these were frameshift or nonsense mutations (Figures 1 and 2). Two patients concomitantly had two types of mutations, respectively (Table 1 ). In addition, we also detected four patients with a microdeletion within chromosome 17q containing NF1 (Table 1; Online Supplementary Figure S2). One patient had both an NF1 mutation and $\mathrm{CN}$ alteration and NF1 alterations were detected in seven $(2.1 \%)$ patients (Figure 1; Table 1). Two 
patients: unique patient number (UPN) 57 and UPN 415 also had a heterozygous deletion. Additionally patient UPN 57 with variant allele frequency (VAF) 0.83 had nonsense mutations in the remaining allele, while UPN 50 with VAF 0.94 had 17q uniparental disomy (UPD) (Online Supplementary Figure S2). UPN 105 and UPN 333 had two or three different $\mathrm{CN}$ regions in $N F 1$, respectively with partially homozygous deletions. Other two patients (UPN 262 and 367) had two types of mutations each. However, it was not clear whether these alterations were monoallelic or bi-allelic (UPN 262, VAF 0.28 and 0.26; UPN 367, VAF 0.28 and 0.08). Thus, we concluded that at least four patients (UPN 50, UPN 57, UPN 105, and UPN 333) had bi-allelic NF1 inactivation. Next, on the basis of the VAF of each mutation, we estimated whether NF1 mutations were somatic or germline. If a mutation is a heterozygous germline mutation, then the VAF is around $0.5{ }^{36} \mathrm{We}$ considered mutations in UPN 262 (VAF 0.28 and 0.26 ) and UPN 367 (VAF 0.28 and 0.08) as somatic mutations. Regarding UPN 50 (VAF 0.94) and UPN 57 (VAF 0.83), it was impossible to predict whether these mutations were somatic or germline because their VAF were high owing to their co-existence with heterozygous deletion or UPD. On the contrary, these two mutations were determined as somatic in the COSMIC v90 (URL: https://cancer.sanger.ac.uk/cosmic). R1241X detected in UPN 57 was previously observed in adult AML and E1561X detected in UPN 50 was previously detected in non-hematological malignancies. ${ }^{37,38}$

PTPN11 mutations were detected in $15(4.6 \%)$ patients (Figure 1). Of these, 14 were located in exon 3 or exon 13, which are known mutation hotspots in AML and JMML (Figure 2). ${ }^{39}$ As previously observed, ${ }^{39}$ codon 76 represented a mutational hot spot (four of 15, 27\%) with three different amino acid substitutions (Figure 2), and 13 of the 15 mutations have been reported as somatic mutations. ${ }^{39 \cdot 41}$ Although the remaining two mutations (V45L and T493I) have not been confirmed as somatic mutations, V45L was earlier detected in lung carcinoma and showed an association with activation of protein-tyrosine phosphatase. ${ }^{41}$ However, T493I has not been reported in any hematological or other disease. These two variants have not been reported as SNP on any database such as COSMIC v90, ClinVar, mutations taster, Ensembl GRCh37, or db SNP (URL: https://www.ncbi.nlm.nih.gov/snp/, https://www.ncbi.nlm.nih.gov/snp/, http://grch37.ensembl.org/index.html, https://www.ncbi.nlm.nih.gov/clinvar/, and http://mutationtaster.org/); therefore, we identified these as novel disease-causing mutations.

$C B L$ mutations were found in six $(1.8 \%)$ patients (Figure 1). Among these, four were deletions or insertions and deletions in exon 8 and two were missense mutations in exon 9. Five of these mutations were in the linker region or the RING finger domain which were previously reported as the affected regions in myeloid malignancies with CBL mutations (Figure 2)..$^{12,13,18}$ None of the six mutations have been reported as SNP or germline mutations in any online databases or previous reports. ${ }^{42} C B L$ mutations especially missense mutations were shown to exhibit a strong association with 11 q-acquired UPD. ${ }^{18} 11 \mathrm{q}$ UPD was detected in only one patient with a missense mutation (UPN 97) by CN analysis (Online Supplementary Figure S2).

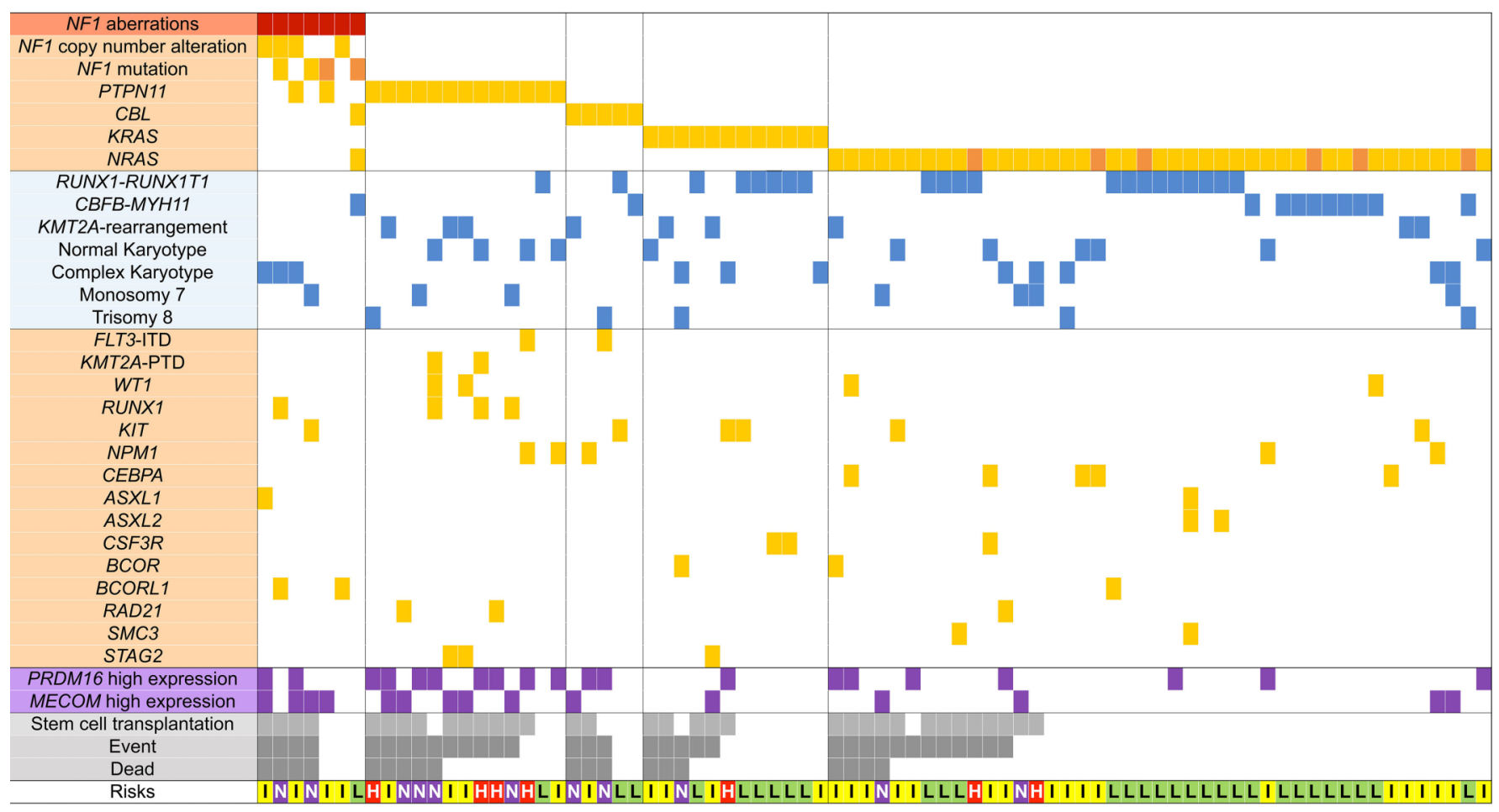

Risks H High risk I Intermediate risk [L Low risk N Non-CR

Two mutations in one patient

Figure 1. Molecular and cytogenetic aberrations in 80 pediatric acute myeloid leukemia patients with RAS pathway alterations. Each column displays the cytogenetic aberration pattern and clinical status of an individual sample. Orange indicates RAS pathway and other genetic alterations. Blue indicates chromosomal aberrations. Purple indicates gene expression. Gray indicates clinical outcome. Blanks indicate the absence of the chromosomal aberration, genetic alteration, or prognostic event. CR: complete remission. 
A

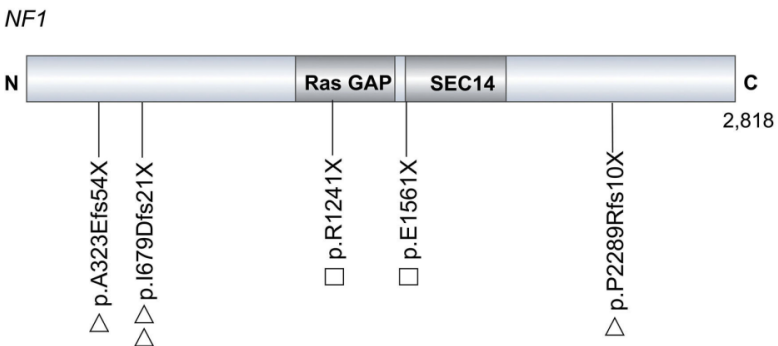

Ras GAP, Ras GTPase-activating protein

C

$C B L$

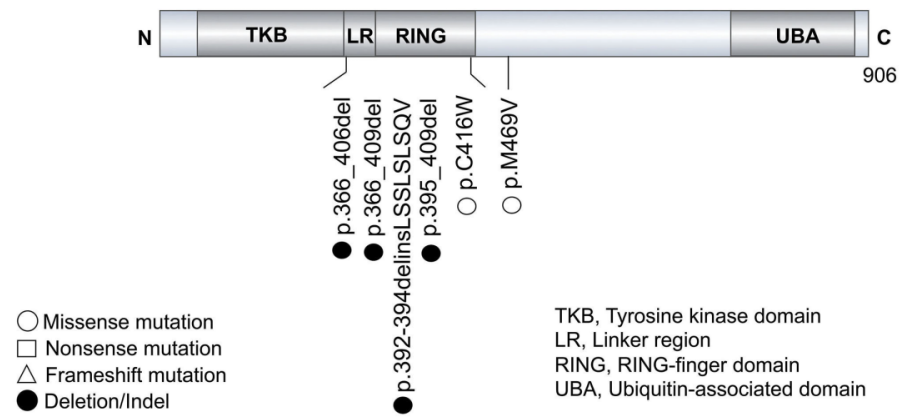

B

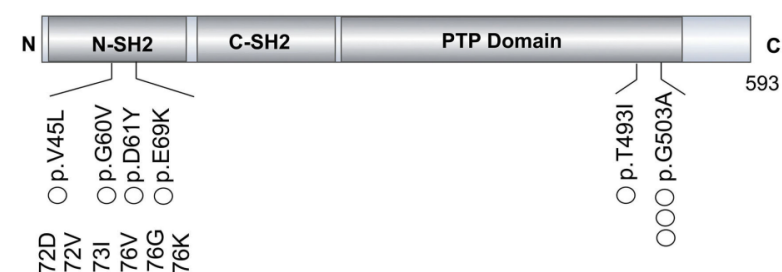

$\mathrm{N}-\mathrm{SH} 2, \mathrm{~N}$-terminal Src homology 2 domain $\mathrm{C}-\mathrm{SH} 2, \mathrm{~S}$-terminal Src homology 2 domain
D

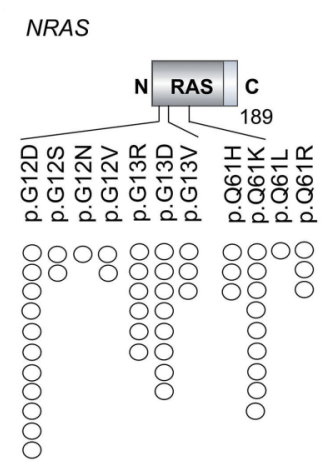

E KRAS

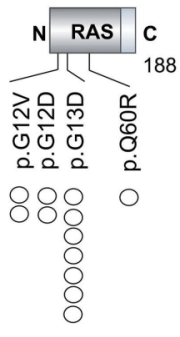

RAS, RAS GTPase family domain

Figure 2. Gene diagrams depicting RAS pathway mutations in pediatric patients with acute myeloid leukemia. (A) NF1 mutations (NCBI reference sequence; NM_000267); (B) PTPN11 mutations (NCBI reference sequence; NM_002834); (C) CBL mutations (NCBI reference sequence; NM_005188); (D) NRAS mutations (NCBI reference sequence; NM_002524); (E) KRAS mutations (NCBI reference sequence; NM_004985).

NRAS and KRAS mutations were detected in 44 (13.4\%) and $12(3.7 \%)$ patients, respectively (Figure 1). All NRAS and KRAS mutations were missense mutations in codon 12, 13, or 61, which are well known hotspots (Figure 2). ${ }^{43}$ Six patients concomitantly had two missense mutations in NRAS.

\section{Clinical and cytogenetic characteristics of patients with RAS pathway alterations}

The clinical characteristics of patients with RAS pathway alterations are summarized in the Online Supplementary Table S4. Patients with RAS pathway alterations showed a significantly higher frequency of detection of monosomy 7 as compared to those without RAS pathway alterations $(P<0.001)$. FLT3-ITD and KIT mutations were significantly less frequent in patients with RAS pathway alterations (FLT3-ITD, $P=0.001$; KIT mutations, $P=0.004)$. Age, sex, or relapse rate were not significantly different between patients with or without each specific RAS pathway alteration.

Patients with $C B L$ mutations had significantly higher WBC count at diagnosis $(P=0.026$; Online Supplementary Table S4; Online Supplementary Figure S3). The frequency of stem cell transplantation (SCT) was significantly higher in patients with PTPN11 mutations $(P=0.035)$, and significantly lower in patients with NRAS mutations $(P=0.022$; Figure 1; Online Supplementary Table S4). PTPN11 mutations were significantly fewer $(P=0.024)$ in patients with low risk, i.e., core binding factor (CBF)-AML, and NRAS mutations were significantly higher $(P=0.017)$ in these patients (Figure 1; Online Supplementary Table S4). The frequency of detection of $N F 1$ alterations was significantly higher in patients with complex karyotype $(P=0.031)$ and $M E C O M$ high expression ( $P=0.013$, Figure 1; Online
Supplementary Table S4). PTPN11 mutations were significantly more frequently detected in patients with monosomy $7(P=0.047), R U N X 1$ mutations $(P=0.004), P R D M 16$ high expression $(P=0.002)$, and $M E C O M$ high expression $(P=0.004)$ (Figure 1; Online Supplementary Table S4). NRAS mutations were frequently detected in inv(16)(p13q22)/CBFB-MYH11 ( $P=0.001)$ and monosomy $7(P=0.013)$. NRAS mutations were also mutually exclusive with FLT3-ITD $(P=0.005)$ and KIT mutations $(P=0.040)$ (Figure 1; Online Supplementary Table S4). Although there was no significant difference, three of six patients with CBL mutations were identified in CBF-AML $(P=0.411)$ (Figure 1; Online Supplementary Table S4).

\section{Prognosis of patients with RAS pathway alterations}

We analyzed the prognosis of patients with or without RAS pathway alterations using the Kaplan-Meier method (Figure 3; Online Supplementary Figure S4). Despite the small sample size, alterations of NF1 and PTPN11 showed a significant association with poor prognosis. Although there was no significant difference in EFS between patients with or without NF1 alterations, the OS of patients with NF1 alterations was significantly worse than that of patients without NF1 alterations (2year OS, $42.9 \%$ vs. $82.3 \%, P=0.003$ ) (Figure $3 \mathrm{~A}$ and $\mathrm{B}$ ). Although no significant differences were observed in OS, PTPN11 mutations were significantly associated with poor EFS (2-year EFS, 30.0\% vs. 59.8\%, $P=0.013$ ) (Figure $3 \mathrm{C}$ and $\mathrm{D})$. The OS and EFS of patients with NRAS mutations were significantly better than those of patients without NRAS mutations (2-year OS, $97.7 \%$ vs. $79.0 \%$, $P=0.014$; 2-year EFS, $74.9 \%$ vs. $55.9 \%, P=0.021$ ) (Figure $3 \mathrm{E}$ and $\mathrm{F}$ ). The presence of $C B L$ or KRAS mutations showed no significant impact on prognosis (Online 
Table 1. Summary of characteristics of pediatric acute myeloid leukemia patients with NF1 alteration.

\begin{tabular}{|c|c|c|c|c|c|c|c|c|c|c|c|c|c|c|c|}
\hline UPN & $\begin{array}{l}\text { Nucleotide } \\
\text { change* }\end{array}$ & $\begin{array}{l}\text { Amino acid } \\
\text { change* }\end{array}$ & VAF & $\begin{array}{c}\text { Copy } \\
\text { number }\end{array}$ & $\begin{array}{l}\text { Start } \\
\text { to end }\end{array}$ & Sex & $\begin{array}{l}\text { Age, } \\
y\end{array}$ & $\begin{array}{l}\text { WBC, } \\
\times 10^{9} / \mathrm{L}\end{array}$ & Cytogenetics & $\begin{array}{l}\text { Additional } \\
\text { genetic } \\
\text { aberrations }\end{array}$ & CR & Relapse & Event & SCT & Prognosis \\
\hline 50 & c.G4681T & p.E1561X & 0.94 & - & - & $\mathrm{M}$ & 13.7 & 19.9 & $45, X Y,-7[13] / 46, X Y[7]$ & KIT & - & - & + & + & Death \\
\hline 367 & $\begin{array}{c}\text { c.966_967insGA } \\
\text { c.2027dupC }\end{array}$ & $\begin{array}{l}\text { p.A323Efs54X } \\
\text { p.I679Dfs21X }\end{array}$ & $\begin{array}{l}0.28 \\
0.08\end{array}$ & - & - & M & 7 & 9.9 & $\begin{array}{c}47, \mathrm{XY},+11[18] / 54 \\
\text { em },+\mathrm{X},+10,+11,+13,+14, \\
+20,+21[1] / 46, \mathrm{XY}[1]\end{array}$ & PTPN11 & + & - & - & - & Alive \\
\hline 57 & c.C3721T & R1241X & 0.83 & $\begin{array}{l}1.16 \\
0.99\end{array}$ & $\begin{array}{c}1225849-29422297 \\
29485961-30325657\end{array}$ & M & 15.2 & 69.0 & $\# 1$ & $\begin{array}{l}\text { RUNXI, } \\
\text { BCORLI }\end{array}$ & - & - & + & + & Death \\
\hline 105 & - & - & - & $\begin{array}{l}1.02 \\
0.29 \\
0.95\end{array}$ & $\begin{array}{l}27009658-29588669 \\
29626467-29679186 \\
29683418-30325657\end{array}$ & M & 10.8 & 15.5 & $\# 2$ & $A S X L 1$ & + & + & + & + & Death \\
\hline 415 & - & - & - & $\begin{array}{l}1.26 \\
1.04\end{array}$ & $\begin{array}{c}1225849-29422297 \\
29485961-30325657\end{array}$ & $\mathrm{~F}$ & 12.3 & 1.9 & $\begin{array}{c}\text { 45,XX,ins(1;?) } \\
\text { (q21;?), add(4)(q12), } \\
\text { add(7)(q36), der(17;18) } \\
\text { (q10;q10)[20] }\end{array}$ & PTPN11 & + & + & + & + & Death \\
\hline
\end{tabular}

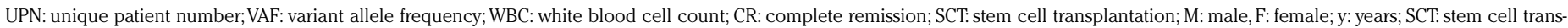
plantation. *NCBI reference sequence; NM_00267.\#1 47,X,-Y,add(3)(q11.2),+6, add(6)(p21)x2,+7,del(8)(q24)der(8)t(1;8)(q11;q24),del(11)(q?),add(17)(p11.2)[7]/48,sl,+22 [6]/47,sl,-14,+mar1 [2]

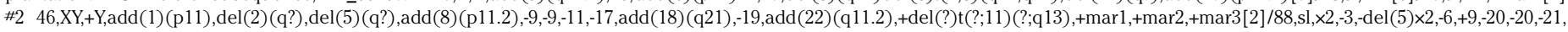
-mar1,-mar3×2,+5mar[1]/47,XY,+Y[9]

Supplementary Figure S4). With respect to prognosis, patients with $C B L$ mutations were divided into two distinct groups based on the presence of CBF. All CBF-AML patients with $C B L$ mutations achieved complete remission and were alive. However, all non-CBF-AML patients relapsed and died (Table 2 ).

Next, we performed multivariate analysis using the Cox regression analysis to determine the prognostic impacts of RAS pathway alterations (Table 3 ). Besides RAS pathway mutations, we used $\mathrm{t}(8 ; 21)(\mathrm{q} 22 ; \mathrm{q} 22) / R U N X 1-R U N X 1 T 1$, CBFB-MYH11, monosomy 7, complex karyotype, FLT3ITD, 5q-, FUS-ERG, NUP98-NSD1, and PRDM16 high expression as explanatory variables in the multivariate analysis; these cytogenetic aberrations were used for risk classification in the AML-05 trials (Online Supplementary Figure S1) or were recently shown to affect the prognosis. ${ }^{33,44}$ Remarkably, NF1 alterations were associated with inferior $O S$ in multivariate analysis (hazard ratio [HR] 4.109; 95\% CI:, 1.471-11.48; $P=0.007]$ (Table 3). In univariate analysis, PTPN11 mutation was associated with inferior EFS (HR 2.142; 95\% CI: 1.157-3.965; $P=0.015$ ) (Table 3). However, PTPN11 mutation was not associated with inferior EFS (HR 1.239; 95\% CI: 0.616-2.494; $P=0.548)$ in multivariate analysis; this indicated that cooccurring aberrations contributed to worse outcomes (Table 3). In multivariate analysis, NRAS mutation was a favorable prognostic factor for both OS and EFS (OS: HR 0.309; 95\% CI: 0.112-0.849; $P=0.023$; EFS: HR, 0.530; 95\% CI: $0.293-0.961 ; P=0.037$ ) (Table 3 ). These results suggested that alterations of NF1 and NRAS were independent predictors of prognosis in pediatric patients with AML. CBFB-MYH11 could not be evaluated accurately for $\mathrm{OS}$ in the Cox regression analysis because 27 patients with $C B F B-M Y H 11$ enrolled in this study were all alive. The OS of patients with $C B F B-M Y H 11$ was significantly better than that of patients without $C B F B-M Y H 11$ in the Kaplan-Meier method $(P=0.005)$. (Online Supplementary Figure S5)

\section{Discussion}

In this study, we detected RAS pathway alterations in $80(24.4 \%)$ of the 328 patients with AML (NF1 [n=7, $2.1 \%$, PTPN11 [n=15, 4.6\%], CBL ( $\mathrm{n}=6,1.8 \%$ ], NRAS $[\mathrm{n}=44,13.4 \%], \operatorname{KRAS}[\mathrm{n}=12,3.7 \%])$. Most of these were mutually exclusive and were also mutually exclusive with aberrations involving other signal transduction pathways such as FLT3-ITD and KIT mutation (Figure 1).

Loss of the wild-type allele of NF1, either through deletions or mutations, has been implicated in the pathogenesis of hematological malignancies. ${ }^{11}$ We have summarized previous reports on NF1 alterations in adult and pediatric AML in the Online Supplementary Table S5. NF1 deletions have been reported in 3.5-10.5\% of adult patients with AML; in addition, $20-50 \%$ of patients with NF1 deletions had concomitant NF1 mutations in the remaining allele. ${ }^{14-16}$ In this study, the frequency of $N F 1$ alterations was less than that in previous reports pertaining to adult patients. In addition, at least four of the seven (57\%) patients with $N F 1$ alterations had bi-allelic NF1 inactivation (Table 1). NF1 alterations have been frequently reported in complex karyotype AML; in addition, NF1 alterations were shown to be associated with poor prognosis in adult AML. ${ }^{3}$ In the contemporary literature, there are few reports about NF1 alteration in pediatric AML. Balgobind et al. detected NF1 deletion in two of the $71 \mathrm{AML}$ patients with KMT2A rearrangement, one of whom experienced relapse. ${ }^{11}$ Consistent with previous reports, NF1 alterations were frequently detected in complex karyotype, and were associated with poor OS in this study (Figure 3; Table 3). None of the four patients with relapse or induction failure were rescued by SCT (Figure 1; Table 1). Our findings suggest that more intensive primary chemotherapy may be an option to rescue AML patients with NF1 alterations including use of novel molecular targeted therapy such as mTOR inhibitors. In a study by Parkin et al., NF1 null blasts showed sensitivity to rapamycin-induced apoptosis.,14 
A

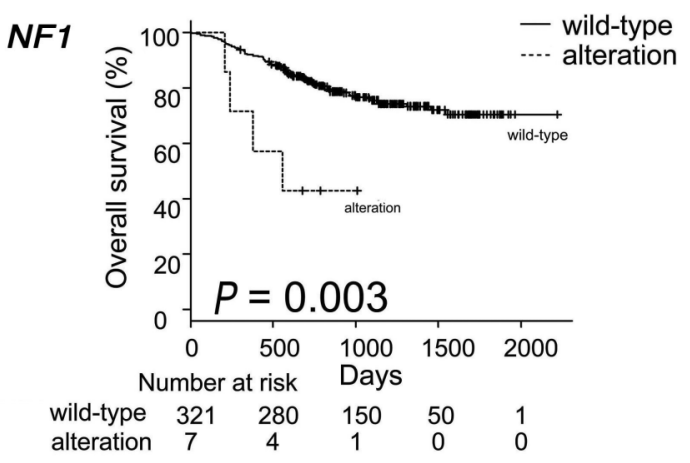

C

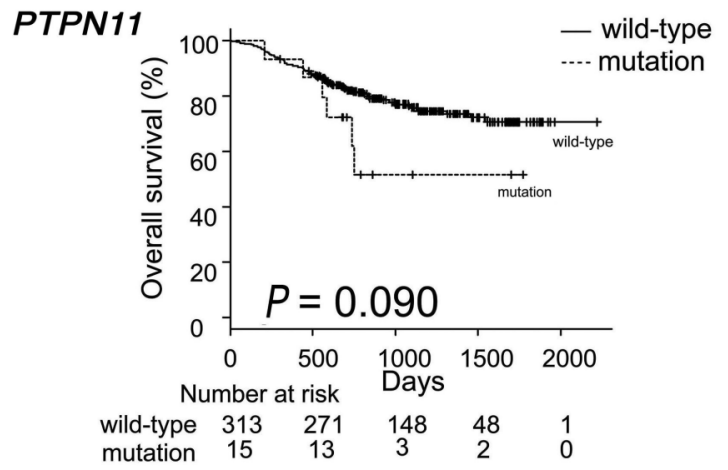

E

\section{NRAS}

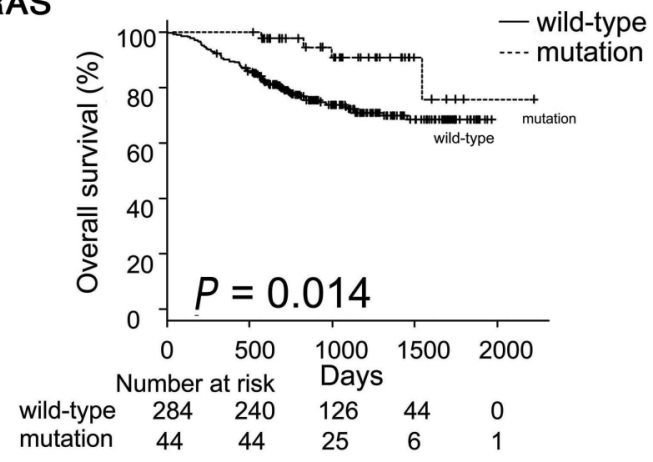

B NF1

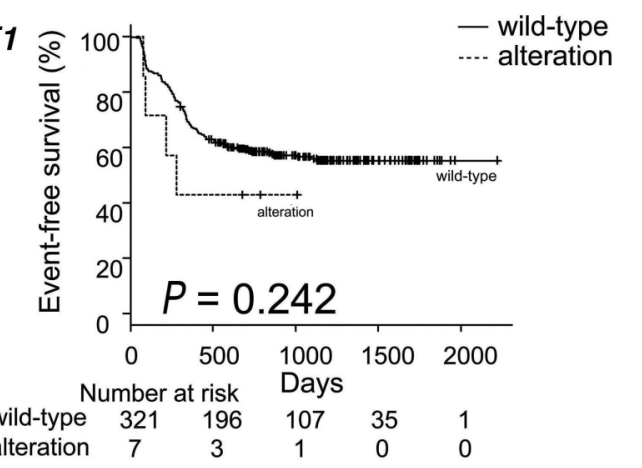

D

PTPN11

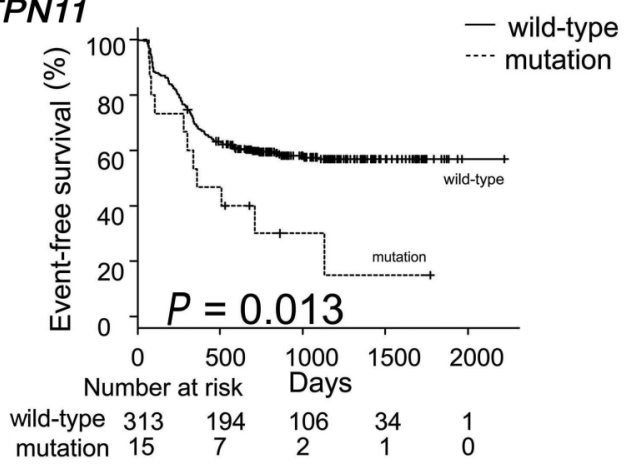

$\mathrm{F}$

NRAS

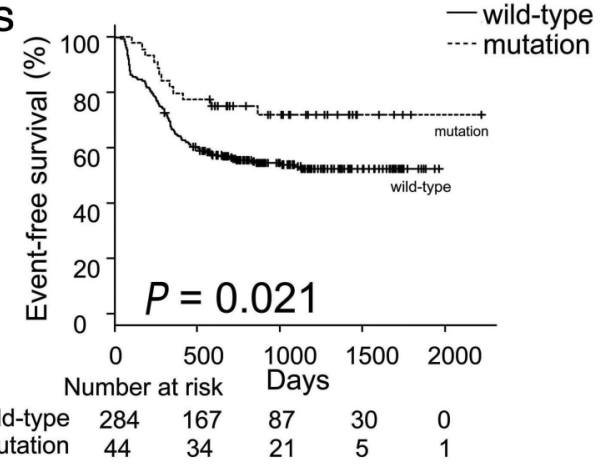

Figure 3. Prognostic significance of NF1, PTPN11, and NRAS alterations in pediatric patients with acute myeloid leukemia. (A), (C), and (E) show Kaplan-Meier curves of overall survival of patients with and without NF1, PTPN11, and NRAS alterations. (B), (D), and (F) show Kaplan-Meier curves of event-free survival of patients with and without NF1, PTPN11, and NRAS alterations.

We also detected 15 PTPN11 mutations including two novel mutations (Table 2). In several previous studies, PTPN11 mutations have been reported to be associated with acute monoblastic leukemia (FAB-M5), ${ }^{4,7}$ however, no such tendency was observed in this study (data was not shown). PTPN11 mutations in our cohort were frequently detected in AML, minimally differentiated (FAB-M0) $(P=0.026)$ and erythroleukemia (FAB-M6) $(P=0.047)$. Goemans et al. also reported that the prevalence of PTPN11 was not increased in acute monoblastic leukemia (FAB-M5) suggesting that differences could exist in the ethnic background of the patients studied..$^{45}$ In a study by Alfayez et al., PTPN11 mutation in adult AML patients was associated with adverse prognosis. ${ }^{4}$ However, the prognostic relevance of PTPN11 has not been reported in pediatric AML. ${ }^{6,7}$ In this study, patients with PTPN11 mutations had a high frequen- cy of RUNX1 mutations, MECOM high expression, and $P R D M 16$ high expression which are strongly associated with poor prognosis (Figure 1; Online Supplementary Table S4)..$^{30,33,46-48}$ In our study, PTPN11 mutations were associated with poor EFS in univariate analysis; however, multivariate analysis revealed no significant impact of PTPN11 mutations on EFS or OS (Figure 3; Table 3). A significantly greater proportion of patients with PTPN11 mutations received SCT (Online Supplementary Table S4); in addition, five of 11 patients with events were rescued by SCT (Figure 1). We consider that AML patients with PTPN11 mutations tended to have a high frequency of relapse or induction failure, and some of these patients were successfully rescued by SCT.

Consistent with a previous report, ${ }^{49}$ NRAS mutations were significantly more frequently detected in $C B F B$ MYH11 (Figure 1; Online Supplementary Table S4). Previous 
Table 2. Summary of characteristics of pediatric acute myleoid leukemia patients with PTPN11 and CBL mutations.

\begin{tabular}{|c|c|c|c|c|c|c|c|c|c|c|c|c|c|}
\hline Gene & UPN & $\begin{array}{l}\text { Nucleotide } \\
\text { change* }\end{array}$ & $\begin{array}{l}\text { Amino acid } \\
\text { change* }\end{array}$ & Sex & $\begin{array}{l}\text { Age, } \\
y\end{array}$ & $\begin{array}{l}\text { WBC, } \\
\times 10^{9} / \mathrm{L}\end{array}$ & Cytogenetics & $\begin{array}{l}\text { Additional } \\
\text { genetic } \\
\text { aberrations }\end{array}$ & CR & Relapse & Event & SCT & Prognosis \\
\hline \multirow[t]{15}{*}{ PTPN11 } & 45 & A227T & $\mathrm{E} 76 \mathrm{~V}$ & $\mathrm{M}$ & 4.8 & 33.9 & $\begin{array}{l}45, \mathrm{XY},-7[1] / 45, \mathrm{sl}, \mathrm{t}(3 ; 12) \\
\text { (q26;p13)[18]/46,XY[1] }\end{array}$ & - & - & + & + & + & Death \\
\hline & 52 & G133C & V45L & $\mathrm{F}$ & 14.1 & 16.5 & $46, \mathrm{XX}[20]$ & WT1, KMT2A-PTD & - & - & + & - & Death \\
\hline & 113 & $\mathrm{C} 215 \mathrm{~T}$ & $\mathrm{~A} 72 \mathrm{~V}$ & $\mathrm{~F}$ & 10.3 & 17.8 & $46, \mathrm{XX}$, add $(12)(\mathrm{p} 11)[12] / 46, \mathrm{XX}[8\}$ & $\begin{array}{l}\text { CBL, KRAS, } \\
\text { KMT2A-ELL, } \\
\text { WT1, STAG2 }\end{array}$ & + & + & + & + & Alive \\
\hline & 127 & C218T & T73I & $\mathrm{F}$ & 0.4 & 17.1 & $47, \mathrm{XX}, \mathrm{t}(7 ; 12)(\mathrm{q} 36 ; \mathrm{p} 13),+19[20]$ & RAD21 & + & + & + & + & Alive \\
\hline & 142 & G1508C & G503A & M & 6.9 & 190.5 & N/A & KMT2A-MLLT3 & + & + & + & + & Death \\
\hline & 156 & $\mathrm{C} 215 \mathrm{~A}$ & A72D & $\mathrm{F}$ & 11.5 & 4.5 & $46, \mathrm{XX}[20]$ & FLT3-ITD, NPMI & + & - & - & + & Alive \\
\hline & 177 & $\mathrm{~A} 227 \mathrm{G}$ & E76G & $\mathrm{M}$ & 2.9 & 25.2 & $\begin{array}{l}45, X Y,-7[1] / 45, \mathrm{sl}, \mathrm{t}(11 ; 21) \\
\quad(\mathrm{q} 13 ; q 22)[19]\end{array}$ & - & - & - & + & + & Death \\
\hline & 249 & G179T & $\mathrm{G} 60 \mathrm{~V}$ & $\mathrm{~F}$ & 11.8 & 60.1 & $46, \mathrm{XX}[20]$ & $\begin{array}{c}N R A S, \text { KMT2A-PTD, } \\
\text { RUNX1 }\end{array}$ & + & + & + & + & Alive \\
\hline & 300 & C1478T & T493I & M & 4.2 & 4.6 & $\begin{array}{c}\text { 46,XY,t(8;21)(q22;q22)[2]/46,sl, } \\
\operatorname{del}(9)(\mathrm{q} ?)[7] / 46, \mathrm{XY}[11]\end{array}$ & - & + & - & - & - & Alive \\
\hline & 367 & G226A & E76K & M & 7 & 9.9 & $\begin{array}{c}47, \mathrm{XY},+11[18] / 54, \mathrm{idem},+\mathrm{X} \\
+10,+11,+13,+14,+20,+21[1] / 46, \mathrm{XY}[1]\end{array}$ & $N F 1$ & + & - & - & - & Alive \\
\hline & 375 & G181T & D61Y & $\mathrm{M}$ & 1.9 & 16.1 & 46,XY,-7,+mar[17]/46,idem,del(6) (q?) [3] & 3] $\quad R U N X 1$ & - & - & + & + & Alive \\
\hline & 415 & G1508C & G503A & $\mathrm{F}$ & 12.3 & 1.9 & $\begin{array}{c}\text { 45,XX,ins(1;?)(q21;?),add(4)(q12) } \\
\operatorname{add}(7)(q 36), \operatorname{der}(17 ; 18)(\mathrm{q} 10 ; q 10)[20]\end{array}$ & $N F 1$ & + & + & + & + & Death \\
\hline & 417 & G1508C & G503A & M & 5.6 & 51.7 & $\begin{array}{c}\text { 46,XY,t(11;19) (q23;p13.1)[17]/ } \\
\text { 47,idem,+8[1]/46,XY[2] }\end{array}$ & $\begin{array}{l}\text { KMT2A-ELL, } \\
\quad \text { STAG2 }\end{array}$ & + & + & + & + & Alive \\
\hline & 425 & G205A & $\mathrm{E} 69 \mathrm{~K}$ & M & 9.8 & 73.2 & $46, \mathrm{XY}[20]$ & NPM1 & + & - & - & - & Alive \\
\hline & 438 & $\mathrm{~A} 227 \mathrm{~T}$ & E76V & $\mathrm{F}$ & 13.6 & 161.0 & $49, \mathrm{XX},+8,+10,+12[20]$ & FUS-ERG & + & + & + & + & Death \\
\hline \multirow[t]{6}{*}{$C B L$} & \multicolumn{2}{|c|}{$\begin{array}{cc}2 & \text { c.1174_1181delins } \\
\text { TTATCATCCTTATCAT } \\
\text { TATCACAGGT }\end{array}$} & $\begin{array}{c}\text { p.392-394 } \\
\text { delins } \\
\text { LSSLSLSQV }\end{array}$ & M & 2.3 & 172.0 & $46, \mathrm{XY}, \mathrm{t}(9 ; 11)(\mathrm{p} 22 ; \mathrm{q} 23)[16] / 46, \mathrm{XY}[4]$ & KMT2A-MLLT3 & - & + & + & + & Death \\
\hline & 67 & c.A1405G & p.M469V & M & 7.4 & 168.1 & $47, \mathrm{XY},+8[20]$ & - & - & + & + & - & Death \\
\hline & 97 & c.T1248G & p.C416W & $\mathrm{F}$ & 11.6 & 38.2 & $47, X X,+18[1] / 46, X X[19]$ & NPM1 & + & + & + & + & Death \\
\hline & 167 & $\begin{array}{l}\text { c.1096-75_1218 } \\
\text { delinsAAGGCT }\end{array}$ & p.366_406del & $\mathrm{M}$ & 9.9 & 20.5 & $\begin{array}{l}\text { 46,XY,t(8;21)(q22;q22)[17]/ } \\
45, \mathrm{X},-\mathrm{Y}, \mathrm{t}(8 ; 21)(\mathrm{q} 22 ; \mathrm{q} 22)[3]\end{array}$ & KIT & + & - & - & - & Alive \\
\hline & 184 & c.1183_1227+27del & p.395_409del & M & 15.1 & 54.2 & 47,XY,inv(16)(p13.1q22),+22[20] & - & + & - & - & - & Alive \\
\hline & $262 \mathrm{c}$ & c.1096-40_1227+35del & p.366_409del & M & 12.3 & 159.3 & 46,XY,inv(16)(p13q22)[20] & $N R A S, N F 1$ & + & - & - & - & Alive \\
\hline
\end{tabular}

UPN: unique patient number;WBC: white blood cell count; CR: complete remission; SCT: stem cell transplantation; N/A: not applicable; M: male; F: female.; y: years. *NCBI reference sequence; PTPN11,NM_002834; CBL, NM_005188.

studies have found inconsistent evidence of the clinical significance of NRAS mutations. ${ }^{8,9}$ In the present study, NRAS mutations were associated with favorable prognosis. This seemed attributable to the characteristics of patients with NRAS mutations, i.e., high frequency of CBFB-MYH11 with no other poor prognostic factors.

11 q-UPD was detected in only one patient with a $C B L$ missense mutation, which might be consistent with a previous study reporting that somatically acquired $C B L$ deletions are frequently heterozygous, whereas most missense mutations are homozygous as a consequence of $11 \mathrm{q}$-UPD..$^{50}$ We summarized previous reports on $C B L$ mutations in AML in the Online Supplementary Table S6. $C B L$ mutations were previously shown to be associated with CBF-AML. ${ }^{13}$ In the present study, three of six patients with CBL mutations had CBF-AML; however, there was no significant association in this respect (Figure 1 , Table 2). Owing to the low incidence of $C B L$ mutation, its prognostic significance is not well characterized. ${ }^{10,12,13}$ Although we did not observe any significant prognostic impact of $C B L$ mutations in our cohort, all three patients without CBF experienced relapse and died (Table 2). These results might suggest that non-CBF patients with CBL mutation show poor prognosis.

RAS pathway alterations are also a major cause of JMML; in addition, each of these alterations are of prognostic relevance in patients with JMML. ${ }^{51,52}$ In previous studies, JMML patients with PTPN11 and NF1 mutations showed significantly poor prognosis. ${ }^{51,52}$ On the other hand, JMML patients with NRAS mutations exhibited favorable outcomes. ${ }^{51,52}$ In our study, the prognostic impact of NF1, PTPN11, and NRAS was similar to that observed in JMML. However, we are unable to explain this similarity because the transformation of JMML to AML is rare.53

There may be some possible limitations in this study. First, we analyzed PTPN11, CBL, NRAS and KRAS mutations by Sanger sequencing because the mutation hotspots of these genes were well known. Although the frequency of these mutations was similar to the previous reports by 
Table 3. Univariate and multivariate Cox regression analyses of overall survival and event-free survival.

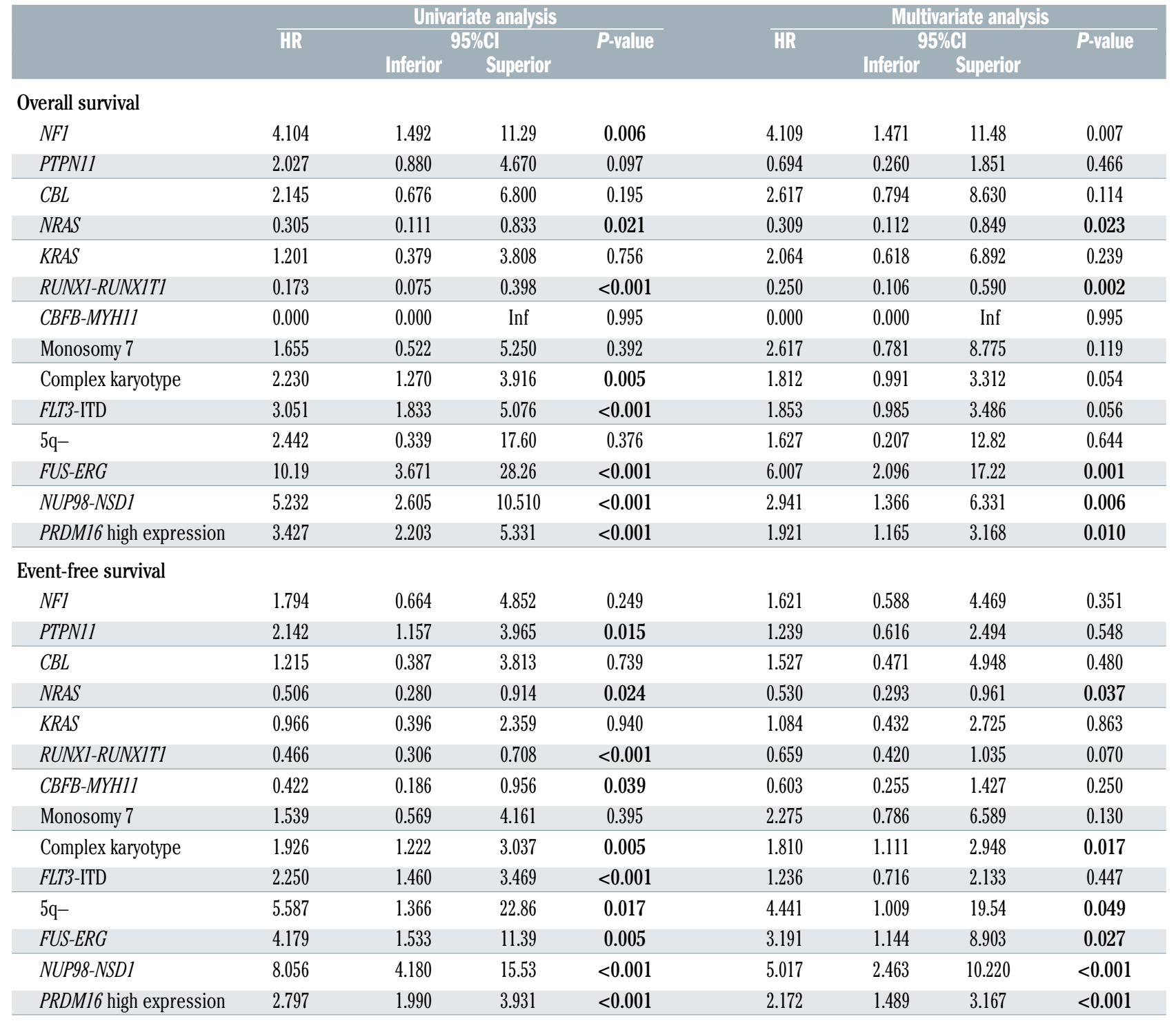

HR: hazard ratio; $\mathrm{Cl}$ : confidence interval.

Sanger sequencing, ${ }^{6.9}$ it appears to be lower than that of the recent pediatric report by targeted deep sequencing. ${ }^{1}$ Next, there were a small number of patients harboring NF1 alterations. Further investigation is needed to determine the clinical significance of NF1 alterations in pediatric AML. Since there have been few reports on NF1 alterations, especially in pediatric AML (Online Supplementary Table S5), our results might be valuable for future analysis. Lastly, we could not analyze germline alterations because of the lack of non-hematopoietic cells. Congenital alterations of RAS pathway genes are known as RASopthies predisposing to hematological malignancies. ${ }^{54}$ Especially for NF1 and CBL, it is difficult to distinguish between somatic and germline mutations because the mutation hotspots overlap. While it is sometimes difficult to diagnose RASopathy because of minor clinical symptoms, patients with distinct clinical features of AML predisposing diseases, such as neurofibromatosis, Noonan syndrome, or CBL syndrome were excluded from the AML-05 trial according to its eligibility criteria. Also, we estimated that most of NF1 and CBL mutations might be somatic from online databases and previous reports. Since there have been few reports of detailed analysis on NF1 and CBL alterations in pediatric AML (Online Supplementary Tables S5 and S6), further analyses are needed.

In conclusion, NF1 alteration is possibly a poor prognostic factor and NRAS mutation is a favorable prognostic factor in pediatric patients with AML. Pediatric AML patients with PTPN11 mutations may show a greater tendency for relapse and induction failure. Detailed analysis of RAS pathway alterations may enable a more accurate prognostic stratification of pediatric AML and may provide novel therapeutic molecular targets related to this signal transduction pathway.

\section{Disclosures}

No conflicts of interest to disclose.

\section{Contributions}

$T K, G Y, N S$, and YH designed and performed the research, analyzed the data, and wrote the paper; YHayashi designed the research, led the project, and wrote the paper; KY, YS, SM, and $S O$ performed the research; KT performed the research and bioinformatics analysis; KO, MS, HA, HM, AS, TTabi, NK, DT, KH, 
TTaga and SA provided patient samples and data. All authors critically reviewed and revised the manuscript.

\section{Acknowledgements}

The authors thank Yuki Hoshino for her valuable assistance in performing the experiments. The authors would like to thank Enago (WWw. enago.jp) for the English language review.

\section{Funding}

This work was supported by a Grant-in-Aid for Scientific
Research on Innovative Areas from the Ministry of Health, Labor and Welfare of Japan (15H05909), a grant for project for development of innovative research on cancer therapeutics ( $P$ DIRECT) from the Japanese Agency for Medical Research and Development (AMED; JP16ck0106064, JP 19ck0106329), the Japanese Society for the Promotion of Science (KAKENHI grants 17K10130, 18H06234, 19K21333, 20K08744), a research grant from the Japanese Society of Hematology, and the Kawano Masanori Memorial Public Interest Incorporated Foundation for Promotion of Pediatrics.

\section{References}

1. Bolouri H, Farrar JE, Triche T Jr, et al. The molecular landscape of pediatric acute myeloid leukemia reveals recurrent structural alterations and age-specific mutational interactions. Nat Med. 2018;24(1):103112

2. Fröhling S, Scholl C, Gilliland DG, Levine RL. Genetics of myeloid malignancies: Pathogenetic and clinical implications. J Clin Oncol. 2005;23(26):6285-6295.

3. Eisfeld AK, Kohlschmidt J, Mrózek K, et al. NF1 mutations are recurrent in adult acute myeloid leukemia and confer poor outcome. Leukemia. 2018;32(12):2536-2545.

4. Alfayez M, Issa GC, Patel KP, et al. The clinical impact of PTPN11 mutations in adults with acute myeloid leukemia. Leukemia. 2021;35(3):691-700.

5. Murakami N, Okuno Y, Yoshida K, et al. Integrated molecular profiling of juvenile myelomonocytic leukemia. Blood. 2018; 131(14):1576-1586

6. Tartaglia M, Martinelli S, Iavarone I, et al. Somatic PTPN11 mutations in childhood acute myeloid leukaemia. Br J Haematol. 2005;129(3):333-339.

7. Loh ML, Reynolds MG, Vattikuti S, et al. PTPN11 mutations in pediatric patients with acute myeloid leukemia: results from the Children's Cancer Group. Leukemia. 2004;18(11):1831-1834

8. Meshinchi S, Stirewalt DL, Alonzo TA, et al. Activating mutations of RTK/ras signal transduction pathway in pediatric acute myeloid leukemia. Blood. 2003;102(4): 1474-1479.

9. Sano H, Shimada A, Taki T, et al. RAS mutations are frequent in FAB type $\mathrm{M} 4$ and M5 of acute myeloid leukemia, and related to late relapse: a study of the Japanese Childhood AML Cooperative Study Group. Int J Hematol. 2012;95(5):509-515.

10. Coenen EA, Driessen EM, Zwaan CM, et al. CBL mutations do not frequently occur in paediatric acute myeloid leukaemia. $\mathrm{Br} \mathrm{J}$ Haematol. 2012;159(5):577-584.

11. Balgobind BV, Van Vlierberghe $\mathrm{P}$, van den Ouweland AM, et al. Leukemia-associated NF1 inactivation in patients with pediatric T-ALL and AML lacking evidence for neurofibromatosis. Blood. 2008;111(8):43224328.

12. Sargin B, Choudhary C, Crosetto N, et al. Flt3-dependent transformation by inactivating $\mathrm{c}-\mathrm{Cbl}$ mutations in AML. Blood. 2007;110(3):1004-1012.

13. Abbas S, Rotmans G, Löwenberg B, Valk PJ. Exon 8 splice site mutations in the gene encoding the E3-ligase CBL are associated with core binding factor acute myeloid leukemias. Haematologica. 2008;93(10): 1595-1597

14. Parkin B, Ouillette P, Wang Y, et al. NF1 inactivation in adult acute myelogenous leukemia. Clin Cancer Res. 2010;16(16): 4135-4137.

15. Boudry-Labis E, Roche-Lestienne C, Nibourel O, et al. Neurofibromatosis-1 gene deletions and mutations in de novo adult acute myeloid leukemia. Am J Hematol. 2013;88(4):306-311.

16. Haferlach C, Grossmann V, Kohlmann A, et al. Deletion of the tumor-suppressor gene NF1 occurs in $5 \%$ of myeloid malignancies and is accompanied by a mutation in the remaining allele in half of the cases. Leukemia. 2012;26(4):834-839.

17. Tartaglia M, Kalidas $K$, Shaw A, et al. PTPN11 mutations in Noonan syndrome: molecular spectrum, genotype-phenotype correlation, and phenotypic heterogeneity. Am J Hum Genet. 2002;70(6):1555-1563.

18. Sanada M, Suzuki T, Shih LY, et al. Gain-offunction of mutated C-CBL tumour suppressor in myeloid neoplasms. Nature. 2009;460(7257):904-908.

19. Matsuo H, Yoshida K, Fukumura K, et al. Recurrent CCND3 mutations in MLL rearranged acute myeloid leukemia. Blood Adv. 2018;2(21):2879-2889.

20. Shimada A, Taki T, Tabuchi K, et al. KIT mutations, and not FLT3 internal tandem duplication, are strongly associated with a poor prognosis in pediatric acute myeloid leukemia with $t(8 ; 21)$ : a study of the Japanese Childhood AML Cooperative Study Group. Blood. 2006;107(5):1806-1809.

21. Döhner K, Schlenk RF, Habdank M, et al H. Mutant nucleophosmin (NPM1) predicts favorable prognosis in younger adults with acute myeloid leukemia and normal cytogenetics: interaction with other gene mutations. Blood. 2005;106(12):3740-3746.

22. Mizushima Y, Taki T, Shimada A, et al. Prognostic significance of the BAALC isoform pattern and CEBPA mutations in pediatric acute myeloid leukemia with normal karyotype: a study by the Japanese Childhood AML Cooperative Study Group. Int J Hematol. 2010;91(5):831-837

23. Sano H, Ohki K, Park MJ, et al. CSF3R and CALR mutations in paediatric myeloid disorders and the association of CSF3R mutations with translocations, including $\mathrm{t}(8 ; 21)$. Br J Haematol. 2015;170(3):391-397.

24. Sano H, Shimada A, Tabuchi K, et al. WT1 mutation in pediatric patients with acute myeloid leukemia: a report from the Japanese Childhood AML Cooperative Study Group. Int J Hematol. 2013;98(4):437-445

25. Yamato G, Shiba N, Yoshida K, et al. ASXL2 mutations are frequently found in pediatric AML patients with $t(8 ; 21)$ RUNX1-RUNX1T1 and associated with a better prognosis. Genes Chromosomes Cancer. 2017;56(5):382-393.

26. Damm F, Chesnais V, Nagata Y, et al.
BCOR and BCORL1 mutations in myelodysplastic syndromes and related disorders. Blood. 2013;122(18):3169-3177.

27. Thol F, Bollin R, Gehlhaar $M$, et al Mutations in the cohesin complex in acute myeloid leukemia: clinical and prognostic implications. Blood. 2014;123(6):914-920.

28. Shiba N, Hasegawa D, Park MJ, et al. CBL mutation in chronic myelomonocytic leukemia secondary to familial platelet disorder with propensity to develop acute myeloid leukemia (FPD/AML). Blood 2012;119(11):2612-2614.

29. Xu F, Taki T, Yang HW, et al. Tandem duplication of the FLT3 gene is found in acute lymphoblastic leukaemia as well as acute myeloid leukaemia but not in myelodysplastic syndrome or juvenile chronic myelogenous leukaemia in children. $\mathrm{Br} \mathrm{J}$ Haematol. 1999;105(1):155-162.

30. Shiba N, Ichikawa H, Taki T, et al. NUP98NSD1 gene fusion and its related gene expression signature are strongly associated with a poor prognosis in pediatric acute myeloid leukemia. Genes Chromosomes Cancer. 2013;52(7):683-693

31. Kong XT, Ida $\mathrm{K}$, Ichikawa $\mathrm{H}$, et al. Consistent detection of TLS/FUS-ERC chimeric transcripts in acute myeloid leukemia with $\mathrm{t}(16 ; 21)(\mathrm{p} 11 ; \mathrm{q} 22)$ and identification of a novel transcript. Blood. 1997;90(3):1192-1199.

32. Balgobind BV, Hollink IH, Reinhardt D, et al. Low frequency of MLL-partial tandem duplications in paediatric acute myeloid leukaemia using MLPA as a novel DNA screenings technique. Eur J Cancer. 2010;46(10):1892-1899.

33. Jo A, Mitani S, Shiba N, et al. High expression of EVI1 and MEL1 is a compelling poor prognostic marker of pediatric AML Leukemia. 2015;29(5):1076-1083.

34. Yoshizato T, Nannya Y, Atsuta Y, et al. Genetic abnormalities in myelodysplasia and secondary acute myeloid leukemia: impact on outcome of stem cell transplantation. Blood. 2017;129(17):2347-2358.

35 . Kanda, Y. Investigation of the freely available easy-to-use software 'EZR' for medical statistics. Bone Marrow Transplant. 2013 48(3):452-458.

36. Smith KS, Yadav VK, Pei S, et al. SomVarIUS: somatic variant identification from unpaired tissue samples. Bioinformatics. 2016;32(6):808-813

37. Papaemmanuil E, Gerstung M, Bullinger L, et al. Genomic classification and prognosis in acute myeloid leukemia. $\mathrm{N}$ Engl J Med. 2016;374(23):2209-2221.

38. Giannakis M, Mu XI, Shukla SA, Genomic correlates of immune-cell Infiltrates in colorectal carcinoma. Cell Rep. 2016;15(4): 857 865

39. Tartaglia M, Niemeyer CM, Fragale A, et al. Somatic mutations in PTPN11 in juvenile 
myelomonocytic leukemia, myelodysplastic syndromes and acute myeloid leukemia. Nat Genet. 2003;34(2):148-150.

40. Tartaglia M, Martinelli S, Cazzaniga G, et al. Genetic evidence for lineage-related and differentiation stage-related contribution of somatic PTPN11 mutations to leukemogenesis in childhood acute leukemia. Blood. 2004;104(2):307-13.

41. Bentires-Alj M, Paez JG, David FS, et al. Activating mutations of the noonan syndrome-associated SHP2/PTPN11 gene in human solid tumors and adult acute myelogenous leukemia. Cancer Res. 2004;64(24): 8816-8820.

42. Martinelli S, Stellacci E, Pannone L, et al. Molecular diversity and associated phenotypic spectrum of germline CBL mutations. Hum Mutat. 2015;36(8):787-796.

43. Bos JL. ras oncogenes in human cancer: a review. Cancer Res. 1989;49(17):4682-4689.

44. Shiba N, Ohki K, Kobayashi T, et al. High PRDM16 expression identifies a prognostic subgroup of pediatric acute myeloid leukaemia correlated to FLT3-ITD, KMT2APTD, and NUP98-NSD1: the results of the Japanese Paediatric Leukaemia/Lymphoma Study Group AML-05 trial. Br J Haematol. 2016;172(4):581-591

45. Goemans BF, Zwaan CM, Martinelli S, et al. Differences in the prevalence of PTPN11 mutations in FAB M5 paediatric acute myeloid leukaemia. $\mathrm{Br} J$ Haematol. 2005;130(5):801-803

46. Döhner H, Estey E, Grimwade D, et al Diagnosis and management of AML in adults: 2017 ELN recommendations from an international expert panel. Blood. 2017;129 (4):424-447.

47. Yamato G, Shiba N, Yoshida K, et al RUNX1 mutations in pediatric acute myeloid leukemia are associated with distinct genetic features and an inferior prognosis. Blood. 2018;131(20):2266-2270.

48. Barjesteh van Waalwijk van DoornKhosrovani S, Erpelinck C, van Putten WL, et al. High EVI1 expression predicts poor survival in acute myeloid leukemia: a study of 319 de novo AML patients. Blood. 2003;101(3):837-845.

49. Goemans BF, Zwaan CM, Miller M, et al. Mutations in KIT and RAS are frequent events in pediatric core-binding factor acute myeloid leukemia. Leukemia. 2005;19(9): 1536-1542.

50. Kales SC, Ryan PE, Nau MM, Lipkowitz S $\mathrm{Cbl}$ and human myeloid neoplasms: the $\mathrm{Cbl}$ oncogene comes of age. Cancer Res. 2010;70(12):4789-4794.

51. Locatelli F, Niemeyer CM. How I treat juvenile myelomonocytic leukemia. Blood. 2015;125(7):1083-1090.

52. Niemeyer CM, Flotho C. Juvenile myelomonocytic leukemia: who's the driver at the wheel? Blood. 2019;133(10):10601070.

53. Luna-Fineman S, Shannon KM, Atwater SK, et al. Myelodysplastic and myeloproliferative disorders of childhood: a study of 167 patients. Blood. 1999;93(2):459-466.

54. Niemeyer CM. RAS diseases in children Haematologica. 2014;99(11):1653-1662. 